\title{
Plasmic Degradation of Crosslinked Fibrin
}

\author{
CHARACTERIZATION OF NEW MACROMOLECULAR SOLUBLE \\ COMPLEXES AND A MODEL OF THEIR STRUCTURE
}

\author{
Charles W. Francis, Victor J. Marder, and Grant H. Barlow, \\ Hematology Unit, Department of Medicine, University of Rochester School \\ of Medicine and Dentistry, Rochester, New York 14642
}

A B S T RACT Crosslinked fibrin was digested by plasmin, and three soluble complexes larger than $\mathrm{DD} / \mathrm{E}$ were purified and characterized. After gel filtration chromatography, the purified complexes were shown to have molecular weights of $465,000,703,000$, and 850,000 , as determined by equilibrium sedimentation. Each of the complexes was dissociated into two or more fragments by SDS-polyacrylamide gel electrophoresis. The structure of these subunit fragments was deduced from determinations of their molecular weights and polypeptide chain composition and from known sites of plasmin cleavage of fibrin. Fragments larger than DD have been identified that contain intact $\gamma \gamma$ crosslinks as well as fragments resulting from cleavages at or near this site. The former include DY (mol wt 247,000), YY (mol wt 285,000), DXD (mol wt 461,000 ), and YXD (mol wt 500,000); and the latter include fragments XD (mol wt 334,000) and XY (mol wt $391,000)$. A schematic model was developed to explain the structure of the large noncovalently bound complexes based on their molecular weight and observed component fragments. Our scheme supports the twostranded half-staggered overlap model as the basic unit of fibrin structure, in which each complex consists of fragments from two adjacent complementary antiparallel fibrin strands. The smallest derivative, complex 1 , is the DD/E complex; complex 2 contains apposed DY and YD fragments, and complex 3 consists of fragments DXD and YY. Complex 4 is less wellcharacterized, but its intact structure is projected to consist of YXD and DXY fragments from adjacent fibrin strands. Each complex is heterogeneous in subunit composition, reflecting additional plasmin cleavages within and/or adjacent to its theoretical boundaries. Since most of the protein initially released into solution from degrading fibrin is as complexes larger than $\mathrm{DD} / \mathrm{E}$,

Received for publication 19 May 1980 and in revised form 8 July 1980. the derivatives described in this report are likely to be major circulating degradation products of crosslinked fibrin in vivo.

\section{INTRODUCTION}

Plasmic degradation of fibrinogen and noncrosslinked fibrin produces transient intermediate fragments $X$ and $Y(1,2)$ and the principal terminal fragments $D$ and E $(3,4)$. Crosslinked fibrin contains Factor XIIIainduced isopeptide bonds between $\gamma$ chains of adjacent monomer pairs that result in major differences of the structure of proteolytic derivatives. The $\gamma \gamma$ crosslink is relatively resistant to plasmin action (5), and extensive degradation of crosslinked fibrin therefore results in the covalently bound dimer, fragment DD (6-8), fragment $D$, and a noncovalently bound complex of DD and E $(9,10)$. Studies of less extensively degraded crosslinked fibrin digests by sodium dodecyl sulfate (SDS)-polyacrylamide gel electrophoresis (11, 12) have identified several derivatives larger than fragment DD, although their molecular weights have not been determined, and possible arrangements into complexes in physiologic conditions have not been considered. Gaffney and Joe (13) have suggested that the DD/E complex may aggregate to form high molecular weight species, but they offered no data to support this hypothesis. Francis et al. (14) have shown that most of the protein initially released into solution from fibrin by plasmic digestion is in noncovalently bound complexes that are larger than DD/E (14). We have now separated and purified three distinct soluble complexes that are much larger than $\mathrm{DD} / \mathrm{E}$. Their molecular weights have been determined by gel elution and by equilibrium sedimentation analysis. The fragments that are dissociated from the complexes by SDSpolyacrylamide gel electrophoresis have been characterized by molecular weight measurements and polypeptide chain analyses. Using these data, we have 
formulated a structure for the macromolecular complexes that is consistent with the half-overlap, twostranded linear fibrin structural model (15-18). Since these derivatives may be protected from degradation in the circulation by proteolytic inhibitors, our results are critical to the development and interpretation of assays to detect circulating degradation products of crosslinked fibrin, whether based upon their size (19-22) or antigenic determinants $(23,24)$.

\section{METHODS}

Human fibrinogen (grade L) was purchased from KABI (AB) (Stockholm). Human thrombin (U. S. standard thrombin, lot H1) and plasmin were kindly supplied by Dr. David Aronson (Bureau of Biologics Standards, Bethesda, Md.). Acrylamide was obtained from Eastman Kodak Co. (Rochester, N. Y.) and recrystallized from a saturated solution of chloroform. Sodium dodecyl sulfate was purchased from Sigma Chemical Co. (St. Louis, Mo.) and recrystallized from ethanol. Phosphorylase A (from rabbit muscle), soybean trypsin inhibitor (type 1-5), carboxypeptidase A (type II), myoglobin (type 1), bovine serum albumin (fraction V), chymotrypsinogen $A$ (type II), and $\beta$-galactosidase (grade IV) were obtained from Sigma Chemical Co. Chromatographically purified human IgM and IgG were purchased from N. L. Cappel Laboratories Inc., Cochranville, $\mathrm{Pa}$. Aldolase, catalase, ferritin, and thyroglobulin were obtained from Pharmacia Fine Chemicals (Div. of Pharmacia, Inc. (Piscataway, N. J.). Epsilon-aminocaproic acid was obtained from Sigma Chemical Co. and aprotinin (Trasylol) from Mobay Chemical Corp. (New York). Crosslinked fibrin was prepared and plasmic degradation of crosslinked fibrin performed as previously described (22). A 50-mg portion of ground fibrin was suspended in $2.0 \mathrm{ml}$ of $0.15 \mathrm{M}$ sodium chloride, $0.05 \mathrm{M}$ Tris-hydrochloric acid buffer, $\mathrm{pH}$ 7.6. Plasmin was added to a final concentration of 0.15 Committee of Thrombolytic Agents U/ml (25), incubation performed at $37^{\circ} \mathrm{C}$ with magnetic stirring, and the reaction terminated with soybean trypsin inhibitor $(0.2 \mathrm{mg} / \mathrm{ml}$ final concentration).

Gel electrophoresis. Discontinuous polyacrylamide gradient gel electrophoresis in nondissociating conditions was performed using a modification (14) of previously described methods $(26,27)$. SDS polyacrylamide gel electrophoresis was performed using gradient gels as previously described $(22,27,28)$. Molecular weights of reduced polypeptide chains were determined by comparison with standards $\beta$-galactosidase $(135,000)$, phosphorylase $A(93,000)$, bovine serum albumin $(68,000), A \alpha(70,000)$ and $B \beta(58,000)$ chains of human fibrinogen, ovalbumin $(43,000)$, carboxypeptidase A $(34,600)$, chymotrypsinogen $(25,700)$, soybean trypsin inhibitor $(21,000)$, and myoglobin $(17,000)$. For nonreduced proteins, molecular weights were estimated by the same method, using as standards fibrinogen $(340,000)$, fragment $X(250,000)$, fragment $Y(150,000)$, fragment $D(100,000)$, bovine serum albumin $(68,000)$, human IgM $(900,000)$, and fibrin dimer $(29)(680,000)$.

Two-dimensional electrophoresis. For nondissociated followed by an SDS-nonreducing system, polyacrylamide gradient gel electrophoresis was performed as above, after which an electrophoretic track was removed and incubated at $37^{\circ} \mathrm{C}$ for $1 \mathrm{~h}$ in a solution of $3.75 \%$ SDS, $0.025 \%$ NaEDTA and $0.1 \mathrm{M}$ boric acid-Tris buffer, $\mathrm{pH}$ 8.6. The strip was then incorporated perpendicularly into the stacking gel of an SDS-polyacrylamide gradient gel and electrophoresed in Tris-borate-SDS buffer as described above. For SDS non- reduced followed by an SDS reduced system, the excised electrophoretic track was incubated at $60^{\circ} \mathrm{C}$ for $1 \mathrm{~h}$ in a solution of $3.75 \%$ SDS, $0.025 \% \mathrm{NaEDTA}$, and $0.15 \mathrm{M}$ dithiothreitol

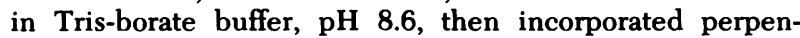
dicularly into the stacking gel, and electrophoresed as above in Tris-borate-SDS buffer, pH 8.6, containing $0.01 \mathrm{M}$ dithiothreitol.

Gel filtration. Plasmic digests of crosslinked fibrin were chromatographed on a column $(158 \times 2.7 \mathrm{~cm})$ of Sephacryl S-300 (Pharmacia Fine Chemicals) using $0.15 \mathrm{M}$ sodium chloride, $0.05 \mathrm{M}$ Tris-buffer, $\mathrm{pH} 7.6$ containing $0.02 \mathrm{M}$ epsilon aminocaproic acid and aprotinin (10 kallikrein inactivator $U /$ $\mathrm{ml}$ final concentration) at a rate of $30 \mathrm{ml} / \mathrm{h}$ at $25^{\circ} \mathrm{C}$. The absorbance at $280 \mathrm{~nm}$ was monitored continuously (UV-2 monitor, Pharmacia Fine Chemicals).

The molecular weights of proteins in the separated peaks were estimated by comparison with the elution positions of proteins of known molecular weight. These included IgM $(900,000)$, thyroglobulin $(669,000)$, ferritin $(440,000)$, catalase $(210,000)$, aldolase $(158,000)$, human immunoglobulin G $(150,000)$, bovine albumin dimer $(136,000)$, and bovine albumin $(68,000)$. Linear regression analysis was used to calculate the best straight line equation for the points describing elution position and the logarithm of molecular weight.

Sedimentation velocity and equilibrium analysis. The sedimentation studies were carried out with the Spinco model $E$ analytical ultracentrifuge (Beckman Instruments, Inc., Spinco Div., Palo Alto, Calif.) equipped with a split beam photoelectric scanning system. The velocity runs were made at $44,000 \mathrm{rpm}$ at or near room temperature in a $12-\mathrm{mm}$ double sector cell. Corrected sedimentation coefficients, $S_{20, w}$, were calculated by standard methods (30). The equilibrium runs were made using a multichannel equilibrium centerpiece at 6,000 and $6,800 \mathrm{rpm}$ at or near $15^{\circ} \mathrm{C}$ using the technique to prevent convection as described by Schachman and Edelstein (31). All scans were made at $280 \mathrm{~nm}$.

The molecular weights were calculated from the equilibrium data by the equation:

$$
\operatorname{mol} w t=\frac{2 R T}{(1-\bar{V} \rho) \omega^{2}} \cdot \frac{d \ln c}{d r^{2}},
$$

where $\mathrm{c}$ is directly proportional to the recorder deflections of the scanner at $280 \mathrm{~nm}$.

\section{RESULTS}

Crosslinked fibrin was exposed to plasmin and the solubilized protein present at different intervals of digestion examined electrophorectically. ${ }^{1}$ Electro-

\footnotetext{
1 The polypeptide chains of fibrinogen and fibrin are named according to the nomenclature suggested by the International Society of Thrombosis and Haemostasis (32). Those of fibrinogen are called $A \alpha, B \beta$, and $\gamma$; those of noncrosslinked fibrin $\alpha, \beta$ and $\gamma$; and those of crosslinked fibrin $\alpha$ polymers $\left(\alpha_{p}\right)$, $\beta$, and $\gamma$ dimers $(\gamma \gamma)$. Fragments of a polypeptide chain resulting from cleavage at the amino terminal end are indicated by a / preceding the symbol of the parent chain, and fragments resulting from cleavage at the carboxy terminal end are indicated by a / following the symbol. Stages of degradation of plasmic lysates of crosslinked fibrin are defined in this report according to Francis et al. (22), based on the SDS-polyacrylamide gel electrophoretic pattern of reduced protein, as shown in Fig. 1C. Stage 1 digests have predominantly intact $\gamma \gamma$ chains. Stage 2 digests have a heterogeneous mixture of $\gamma \gamma$
} 


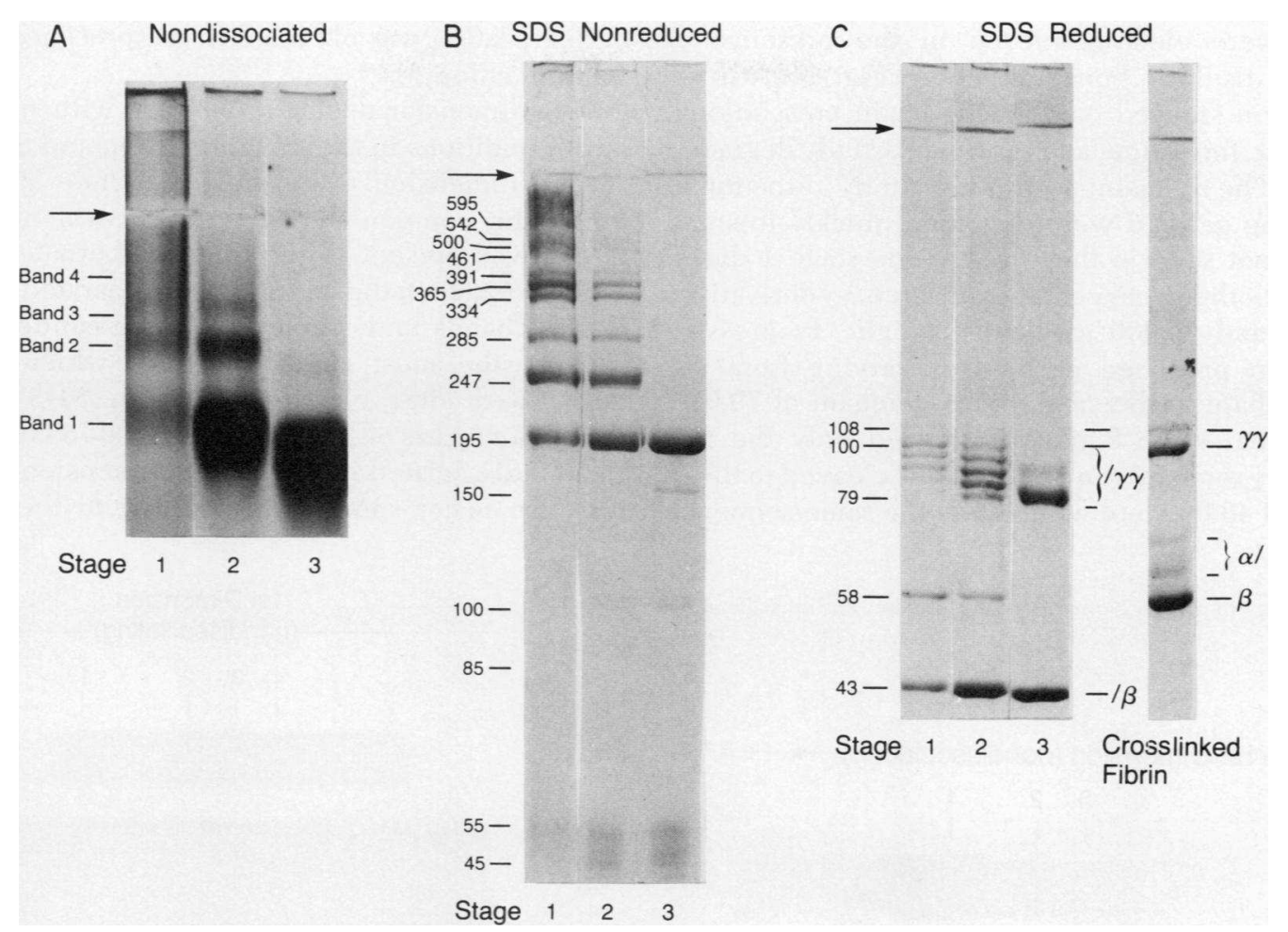

FIgURE 1 Electrophoresis of plasmic digests of crosslinked fibrin in three different polyacrylamide gel systems. In this experiment the stage 1,2 , and 3 digests were exposed to plasmin for 1,4 , and $12 \mathrm{~h}$, respectively. The arrows indicate the demarcation between stacking gel (above) and separating gel (below). The nondissociating, discontinuous buffer system (left) utilized a 4-10\% linear gradient separating gel and a 4\% stacking gel. The amount of protein applied was $30 \mu \mathrm{g}$ in $10 \mu \mathrm{l}$ of buffer for each sample. Electrophoresis in SDS-containing buffer (center) utilized a 4-10\% linear gradient separating gel with a $4 \%$ stacking gel. Amounts of protein applied were 7,9 , and $10 \mu \mathrm{g}$ for the stage 1,2 , and 3 digests, respectively. Disulfide bond reduced protein was electrophoresed in SDS in a 5-14\% linear gradient gel with a 5\% stacking gel (right). Volumes of $20 \mu \mathrm{l}$ containing 7, 17, and $26 \mu \mathrm{g}$ of the stage 1,2 , and 3 samples, respectively, were applied. The crosslinked fibrin used as substrate for these digestions is shown to the far right, and demonstrates $\gamma \gamma$ chains of 100,000 and 108,000 mol wt (34). The bands corresponding to partially degraded forms of the smaller $\gamma \gamma$ chain seen in the stage 1 and stage 2 digests are of $95,000,90,000$ and $86,000 \mathrm{~mol} w \mathrm{wt}$. The terminal $\gamma \gamma$ derivatives seen in the stage 3 digest are of 79,000 and $87,000 \mathrm{~mol}$ wt.

phoresis in nondissociating conditions identified at least four distinct protein stained bands, labeled bands 1-4 in order of decreasing electrophoretic mobility; some protein failed to enter the $4 \%$ stacking gel (Fig. 1A). With longer times of exposure to plasmin, the digest showed progressive loss of bands $2-4$ and of the protein that stayed at the top of the gel, while there was accumulation of protein migrating as band 1 . The terminal digest consisted almost entirely of band 1, and showed no change in electrophoretic pattern even after an additional $24 \mathrm{~h}$ of exposure to enzyme.

chains of molecular size ranging from that of the intact form to that of the smallest remnant. Stage 3 digests have only the smallest $\gamma \gamma$ remnants. Fragments $\mathrm{X}, \mathrm{Y}, \mathrm{D}$, and $\mathrm{E}$ are defined according to Marder and Budzynski (33).
Electrophoresis in the presence of SDS disrupted noncovalent bonds holding the complexes together and demonstrated the covalently bound fragments contained in the digests (Fig. 1B). At least 10 prominent bands with molecular weights between 195,000 and 595,000 and some protein that failed to enter the stacking gel (molecular weight greater than one million) were present in the initial lysate (stage 1). Continued exposure to plasmin resulted in loss of the higher molecular weight bands and increased prominence of the 195,000 molecular weight band, which represented the major derivative in the stage 3 digest. Faintly staining bands with molecular weights of $150,000,100,000$, 85,000 , and $45-55,000$ were also present in each digest.

The same digests and the crosslinked fibrin starting 
material were electrophoresed in the presence of SDS after disulfide bond reduction (Fig. 1C). Crosslinked fibrin showed complete $\gamma$ chain crosslinking, extensive $\alpha_{\mathrm{p}}$ formation, and residual partially degraded $\alpha /$ chains. The $\alpha_{\mathrm{p}}$ chains were seen faintly at the top of the stacking gel and were degraded quickly to small peptides (not seen in these gels). The stage 1 digest contained both intact $\gamma \gamma$ chains and large $/ \gamma \gamma$ derivatives of 95,000 and 90,000 molecular weight. Progressive degradation produced derivatives varying from the intact $\gamma \gamma$ chain to the smallest $/ \gamma \gamma$ remnant of 79,000 . The terminal stage 3 pattern showed only the two smallest $/ \gamma \gamma$ remnants and all $\beta$ chains cleaved to the $/ \beta$ remnant of 43,000 mol wt. Exactly the same sequence of degradation was obtained in the presence of $5 \mathrm{mM}$ calcium chloride.

Two-dimensional electrophoresis with nondissociating conditions in the first dimension and SDS in the second dimension was used to separate and characterize the components of the noncovalently bound derivatives present in the digest supernates (Fig. 2). The 6-h (stage 2) digest supernate separated into three distinct bands under nondissociating conditions (Fig. 2, left), the most anodal band of which (band 1) dissociated after electrophoresis in SDS into two component parts of 195,000 and 45,000-55,000 mol wt. Band 2 dissociated in the second dimension with SDS into two major spots corresponding to the bands of

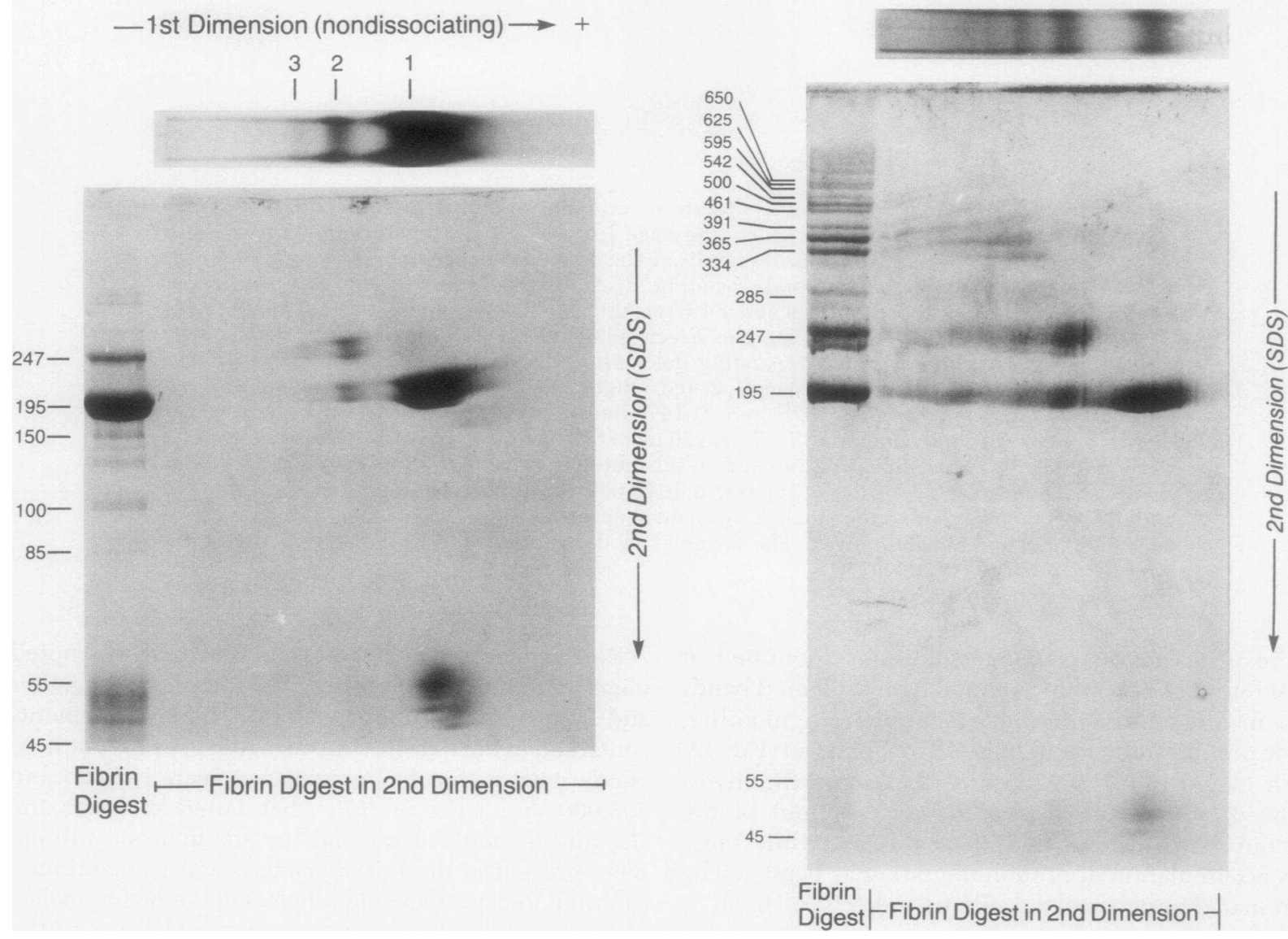

FIGURE 2 Two-dimensional gel electrophoresis of plasmic digests of crosslinked fibrin. Initial separation in a nondissociating buffer using a 3-12\% polyacrylamide gradient gel is shown at the top of both panels; migration in the second dimension proceeded in the presence of an SDS-

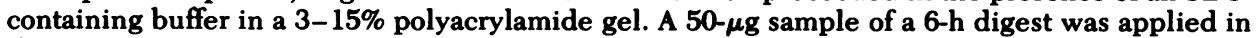
the first panel and a 10- $\mu \mathrm{g}$ sample of the 1-h digest was applied in the second panel. The vertical electrophoresis of the samples labeled "fibrin digest" represents single dimension electrophoresis of the starting material in the SDS-containing buffer. 
195,000 and 247,000 (Fig. 1B). More information on the content of these bands after migration in SDS buffer is available in Fig. 2, right, which shows the results with a 1-h (stage 1) digest. This sample had a greater proportion of bands 2 and 3 and their component parts were more readily visible after dissociation by SDS. In addition to the 195,000 and 247,000 components, band 2 had small amounts of the 45-55,000 moieties. Band 3 resolved into several components, namely those present in bands 1 and 2, plus higher molecular weight bands of 285,000, 334,000, 365,000, and 391,000 (Fig. 1B). If present, bands of $45-55,000$ were too faint to be visible in the band 3 pattern. The remaining bands seen in the first dimension were poorly delineated, and their component parts in the second dimension could not be precisely determined. However, only small amounts of the 195,000 and 247,000 components were present, with relatively larger amounts of the higher molecular weight components, including definite contributions of derivatives with mol wt $>400,000$. SDS-polyacrylamide gel electrophoresis of the chromatographically separated complexes confirmed the results of the two-dimensional electrophoresis.

Two-dimensional electrophoresis using an SDScontaining buffer in the first dimension and disulfide bond reduction in the second dimension determined the polypeptide chain composition of component fragments (Fig. 3). After reduction the 195,000 band showed at least four $\gamma \gamma$ bands, with molecular weights from 79,000 to 95,000 and a / $\beta$ band of 43,000 ; no intact $\beta$ chains were present. The band corresponding to mol wt 247,000 also showed heterogeneity of $\gamma \gamma$ chain composition, but had none of the 79,000 moiety and relatively more of the 90,000 and 95,000 moieties. Most of the $\beta$ chain component was degraded to the 43,000 remnant, but intact $\beta$ chain was also present. The 285,000 band had even less of the smaller $/ \gamma \gamma$ derivatives and a greater proportion of the largest remnant of 95,000; equal proportions of intact $\beta$ and $/ \beta$ chain remnant were present. This trend of increasing molecular size of the $/ \gamma \gamma$ remnant and increasing proportions of intact $\beta$ chain noted in the $195,000,247,000$, and 285,000 bands did not hold for the next larger band of 334,000 , which had the same relative polypeptide chain composition as the 247,000 fragment (Table I). The chains of the 365,000 fragment resembled those of the 334,000 fragment, except for the additional presence of intact $\gamma \gamma$ chains. Just as was noted in the comparison of 247,000 and 285,000 bands, there was a change toward larger polypeptide chains between the patterns of the 334,000 and 391,000 bands. The 391,000 fragment had only the intact $\gamma$ dimer and the largest $/ \gamma \gamma$ remnant of 95,000 , without the smaller $/ \gamma \gamma$ remnants and relatively equal proportions of intact $\beta$ and $/ \beta$ chains. The polypeptide chain components of higher molecular weight fragments were less well resolved

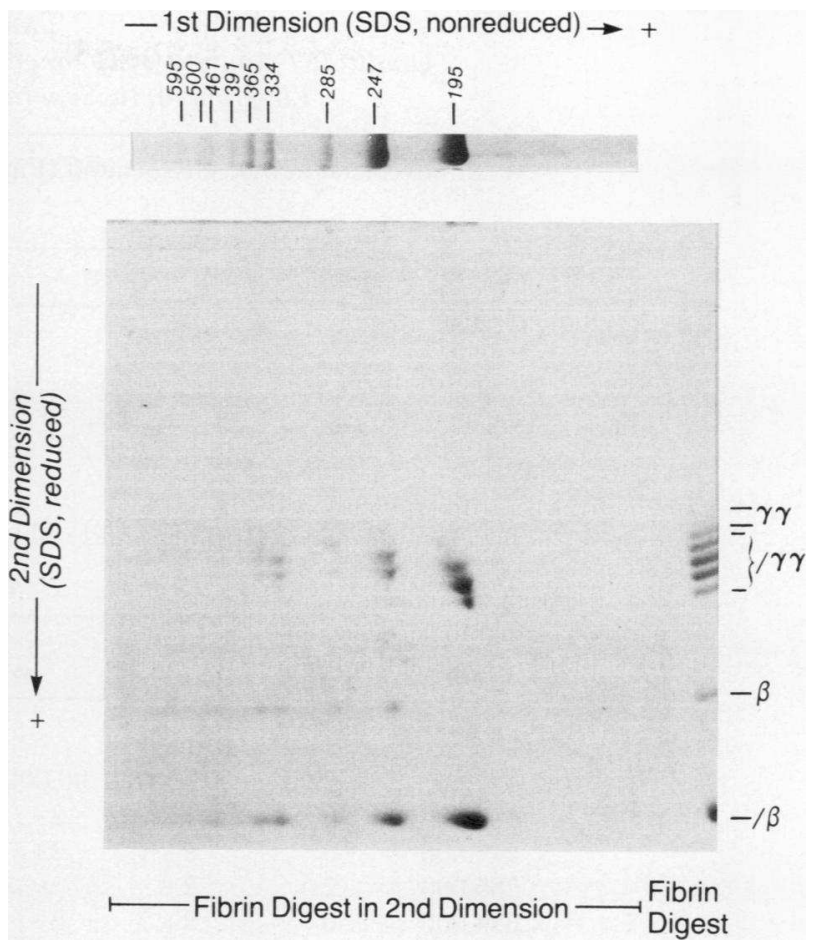

Figure 3 Two-dimensional electrophoresis of the 2-h plasmic digest of crosslinked fibrin. Electrophoresis of the $30-\mu \mathrm{g}$ sample in the first dimension (top gel) was performed in SDS-containing buffer in a 4-10\% polyacrylamide gel, second dimension in reducing buffer in a 5-20\% polyacrylamide gradient gel. The sample labeled "fibrin digest" at the right side of the vertical portion represents electrophoresis of the disulfide-reduced starting material in the second dimension only.

in the gel patterns but appeared to have components similar to those of the 285,000 and 391,000 molecular weight fragments, with predominantly intact $\gamma \gamma$ and the largest of the $/ \gamma \gamma$ chain remnants, none of the smallest I $\gamma \gamma$ remnants, and approximately equal proportions of intact $\beta$ and $/ \beta$ chains.

Gel filtration chromatography on Sephacryl S-300 separated the stage 1 digest of crosslinked fibrin into four protein peaks (Fig. 4), each of which contained one of the corresponding four bands identified by electrophoresis in nondissociating conditions (Fig. 1C). A standard curve correlating elution position with the molecular weight of standard proteins was used to estimate the molecular weights of protein eluting in the four peaks (Table II). Separated protein peaks representing bands 1-4 were immediately subjected to analytical ultracentrifugation without prior concentration or dialysis. The molecular weight plots of this data showed linearity from the top to the bottom of the cell for all samples, indicating that they were homogeneous. The sedimentation coefficients and sedimentation equilibrium molecular weight measure- 
TABLE I

()ualitative Analysis of Component Parts, after Tuo-dimensional Electrophoretic Separations of Fibrin Digests*

\begin{tabular}{|c|c|c|c|c|c|c|c|c|}
\hline \multirow[b]{2}{*}{ Band } & \multicolumn{8}{|c|}{ Fragment } \\
\hline & $45-5.5$ & 195 & 247 & 28.5 & 334 & 365 & 391 & 461 \\
\hline & \multicolumn{8}{|c|}{ molecular weight $\times 10^{-3}$} \\
\hline 1 & $3+$ & $4+$ & 0 & 0 & 0 & 0 & 0 & 0 \\
\hline 2 & $1+$ & $2+$ & $3+$ & 0 & 0 & 0 & 0 & 0 \\
\hline 3 & \pm & $1+$ & $1+$ & \pm & $1+$ & $1+$ & \pm & \pm \\
\hline
\end{tabular}

B SDS-dissociated/disulfide bond reduced (Fig. 3)

\begin{tabular}{cccccccc} 
& \multicolumn{8}{c}{ Polypeptide chain } \\
\cline { 2 - 8 } Fragment & Intact $\gamma \gamma$ & \multicolumn{7}{c}{$/ \gamma$ remnant } & & Intact $\beta$ & $\begin{array}{c}/ \beta \\
\text { remnant }\end{array}$ \\
\hline mol ut & & & & & & & \\
& 100,000 & 95,000 & 90,000 & 86,000 & 79,000 & 58,000 & 43,000 \\
195,000 & 0 & $1+$ & $2+$ & $4+$ & $3+$ & 0 & $4+$ \\
247,000 & 0 & $2+$ & $2+$ & $2+$ & 0 & $1+$ & $3+$ \\
285,000 & $2+$ & $2+$ & \pm & 0 & 0 & $2+$ & $2+$ \\
334,000 & 0 & $2+$ & $2+$ & $1+$ & 0 & $1+$ & $3+$ \\
365,000 & $1+$ & $1+$ & $1+$ & $1+$ & 0 & $1+$ & $2+$ \\
391,000 & $1+$ & $1+$ & \pm & \pm & 0 & $1+$ & $1+$ \\
\hline
\end{tabular}

* Primary data for part A shown in Fig. 2, that for part B in Fig. 3; interpretation based upon arbitrary designations of 0 (absent) to $4+$ (heavy band) for the Coomassie blue-stained gel patterns. Second dimension components of band 4 (Fig. 2) or of fragments of $461,000 \mathrm{~mol}$ wt or greater (Fig. 3) were not sufficiently delineated to allow such analysis.

ments were compared with those estimated by gel exclusion calculations in Table II.

\section{DISCUSSION}

We have shown previously (14) that the soluble complexes released from digesting fibrin always resemble the stage 1 digest, regardless of the exposure time of fibrin to plasmin, and that further degradation occurs primarily in solution after liberation of complexes from the clot. Whereas such degradation to terminal digests occurs in vitro where the solubilized protein is continually exposed to enzyme, it may not occur in vivo where proteolytic inhibitors are present.

In this study we have purified by gel filtration the early complexes released from degrading crosslinked fibrin. Each complex dissociated into two or more covalently bound fragments that could be separated by SDS-polyacrylamide gel electrophoresis (Fig. 2). The smallest complex of $228,000 \mathrm{~mol}$ wt was DD/E (35), but the majority of protein in the stage 1 digest consisted of the previously uncharacterized larger complexes. To understand the structure of the non- covalently bound complexes, we first identified and characterized their covalently bound subunits. After this, we were able to develop a model to show the association of the subunit fragments in complexes and to explain their derivation from the fibrin matrix.

SDS-polyacrylamide gel electrophoresis of unfractionated digests of crosslinked fibrin (Fig. 1B) demonstrates fragment DD (molecular weight 195,000), which is present in all digests but predominates in the stage 3 digest, fragment $\mathrm{Y}(150,000)$, two forms of fragment D $(85,000$ and 100,000$)$, and several forms of fragment E $(45,000$ to 55,000$)$. These fragments have been well characterized, but those of higher molecular weight have not. Alkjaersig et al. (11) found a similar electrophoretic pattern, but they did not determine molecular weights of the individual fragments. Reganon et al. (12) separated plasmic digests of crosslinked fibrin by gel filtration in the presence of SDS and noted two fragments larger than DD, identified as YD with a molecular weight of 220,000 and YY with a molecular weight of 270,000 , by analysis of their reduced polypeptide chain composition.

We have deduced the structure of five fragments 


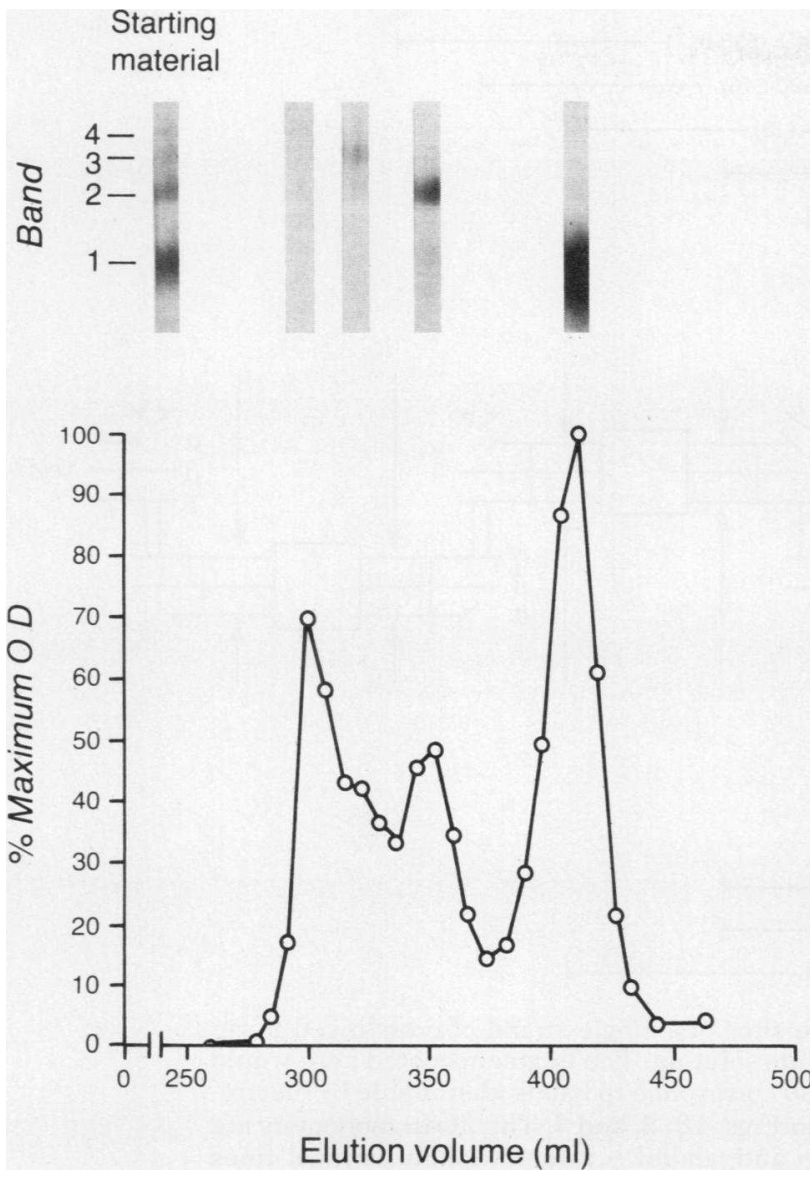

Figure 4 Sephacryl S-300 gel elution of a stage l digest of crosslinked fibrin. Horizontal axis indicates the cumulative elution volume and the vertical axis indicates optical density, normalized for the maximum of 0.44 . The void volume was 285 $\mathrm{ml}$ as determined by elution of dextran blue. Gel electrophoresis of starting material and the protein in each peak under nondissociating conditions shows bands corresponding to those in Fig. 1A. Band 4 is quite faint, and protein in peak 4 was difficult to resolve with this electrophoretic system, some remaining at the top of the stacking gel and some entering only a short distance.

larger than DD by determination of their molecular weights (Fig. 1) and polypeptide chain composition (Fig. 3) and by predictions based on known sites of plasmic cleavage of fibrin. Fig. 5 shows diagrammatically the covalently bound derivatives which would result from cleavages at known sites of plasmin action on fibrin $(33,38-44)$, especially those between the $D$ and $E$ domains and at or near the $\gamma \gamma$ crosslink site. Takagi and Doolittle (45) have identified a plasmin-sensitive site at lysine (405)-glutamine (406) of the $\gamma$ chain that is compatible with the latter, and Olexa et al. (46) and Purves et al. (47) have shown plasmic cleavages near the $\gamma \gamma$ crosslink. The number and molecular weights of fragments predicted were in strong agreement with those identified in digests of crosslinked
TABLE II

Molecular Weight of Nondissociated Complexes in Crosslinked Fibrin Digests*

\begin{tabular}{ccrc}
\hline & & \multicolumn{2}{c}{ Ultracentrifuge } \\
\cline { 3 - 4 } Complex & Gel filtration & $S_{20 . * r}$ & Equilibrium sedimentation \\
\hline 1 & 290,000 & 7.1 & 228,000 \\
2 & 543,000 & 12.3 & 465,000 \\
3 & 747,000 & 16.0 & 703,000 \\
4 & 880,000 & 19.9 & 850,000 \\
\hline
\end{tabular}

* Complexes named according to the elution peaks from Sephacryl S-300 column (Fig. 4) each of which corresponds to bands 1-4 noted in Figs. 1A, 2, and 4.

fibrin (Fig. 1B). In addition, their polypeptide chain composition demonstrated by two-dimensional electrophoresis (Fig. 3, Table I) was consistent with that predicted from the schematic diagram.

The molecular weights predicted for fragment $\mathrm{X}$ or DY were very close, and both were compatible with the band of $247,000 \mathrm{~mol}$ wt (Fig. 1B). The polypeptide chain composition of this fragment identified by twodimensional electrophoresis (Fig. 3, Table I) was compatible only with fragment DY, since crosslinked $\gamma$ chain derivatives would not exist within the structure of fragment $X(42,43,46)$. Since one $D$ to $E$ domain connection remains uncleaved in DY, intact $\beta$ chains as well as / $\beta$ chains should have been present and larger $/ \gamma \gamma$ chains should have predominated; these results were demonstrated in Fig. 3. A fragment corresponding to that of fragment $X$ could not be identified with certainty in the SDS gel electrophoretic patterns, although the occasional splitting of the 247,000 band (Fig. 2, left) may represent both the DY and X fragments.

The structure of fragment YY predicts that it should contain intact $\gamma \gamma$ and $\beta$ chains and this was confirmed by two-dimensional electrophoresis (Fig. 3, Table I). The presence of a $/ \beta$ band is explained by the usual occurrence of $\beta$ chain cleavage before $\gamma$ chain cleavage (44), which would result in some molecules containing cleaved $\beta$ but intact $\gamma-\gamma$ chains. The formation of fragments $\mathrm{XD}$ and $\mathrm{XY}$ would require a cleavage near an isopeptide $\gamma \gamma$ crosslink (Fig. 5). Three bands of 391,000, 365,000 , and $334,000 \mathrm{~mol}$ wt were distinguishable in Fig. 1B, all of which are compatible with only the $\mathrm{XY}$ and XD moieties. That these derivatives were identified suggests that the protection from plasmic degradation that is ascribed to $\gamma$ chain crosslinking (42) may not be complete.

The polypeptide chain composition of higher molecular weight fragments was less well resolved by two-dimensional electrophoresis, but there was excellent agreement between the number of derivatives, their molecular weights predicted from Fig. 5, 

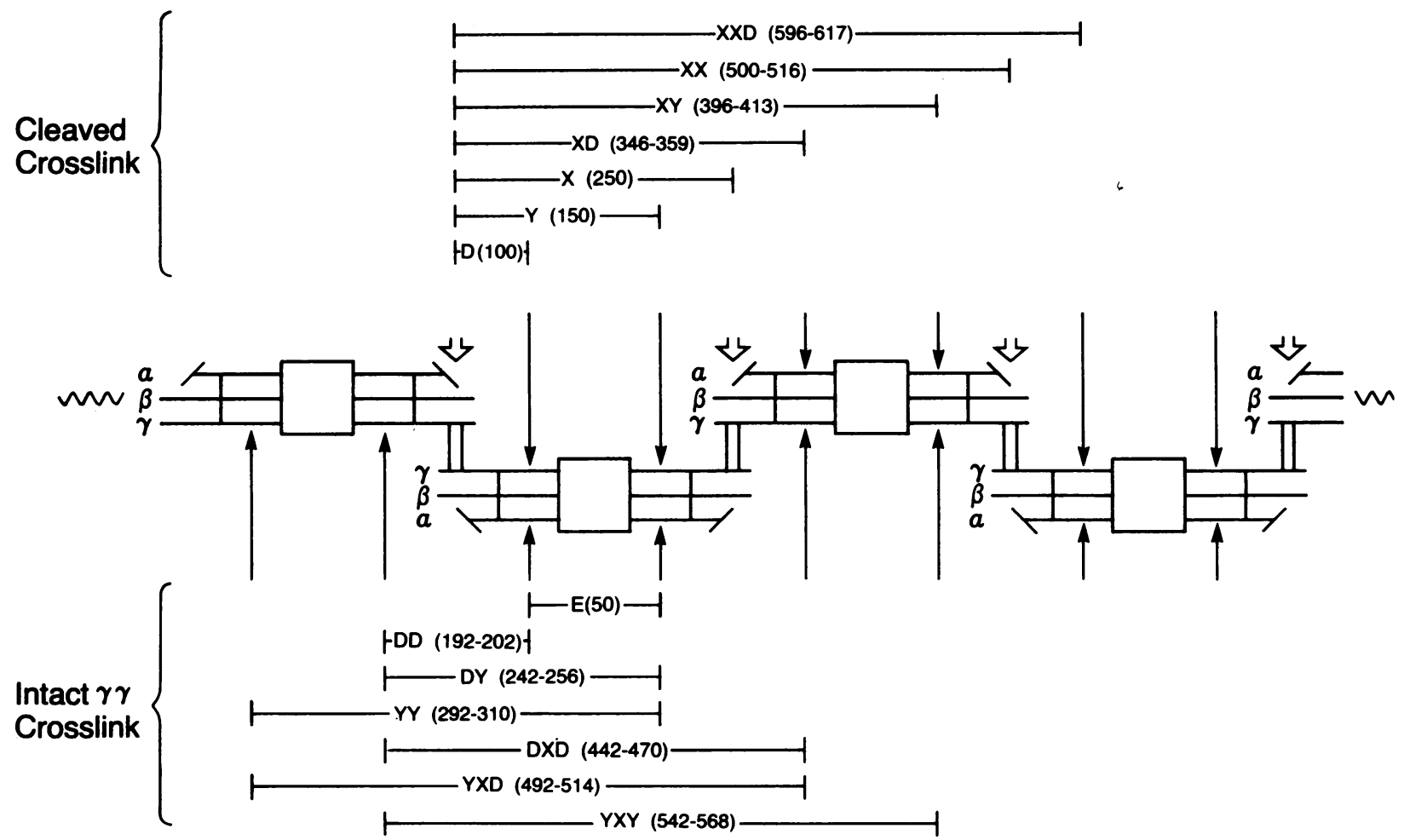

FIGURE 5 Schematic diagram of plasmic cleavage sites of a single strand of crosslinked fibrin and the deduced degradation fragments liberated into solution. The fragments noted here would consist of nonreduced, covalently bound protein and correspond to bands identifiable by electrophoresis in SDS-containing buffers, such as seen in Figs. 1B, 2, and 3. The fibrin monomers are joined by covalent isopeptide crosslinks between antiparallel $\gamma$ chains (double vertical lines (36). Crosslinks between $\alpha$ chains are not shown because $\alpha$ polymer chains are cleaved by plasmin before there is significant proteolysis of $\beta$ or $\gamma$ chains (37). The heavy vertical arrows indicate cleavages of $\gamma$ chains at or near the $\gamma \gamma$ cross-link. Disulfide bond regions are indicated schematically by the square located at the center of each fibrin molecule ( $\mathrm{E}$ domain) and by the single vertical lines joining the lateral extensions of $\alpha, \beta$, and $\gamma$ chains (D domains). Cleavage sites between $\mathrm{D}$ and $\mathrm{E}$ domains are indicated by the thin arrows above and below the fibrin chain. Derivatives that result from cleavages only at these sites and contain an intact $\gamma \gamma$ crosslink are shown in the lower part of the figure ("intact crosslink"). Derivatives that result from both D/E domain cleavages and from cleavages near the $\gamma \gamma$ isopeptide bonds are shown in the upper portion ("cleaved crosslink"). The predicted molecular weight range for each species is noted in parentheses, determined by summation of the molecular weights of constituent, $\alpha, \beta$, and $\gamma$ chain remnants resulting from cleavages at the indicated sites.

and those identified by SDS-polyacrylamide gel electrophoresis (Fig. 1B and Fig. 2, left). Thus, fragments of molecular weight $461,000,500,000,542,000$, and 595,000 correspond to the molecular weights predicted for fragments DXD, YXD or XX, YXY, and XXD, respectively.

The structure of noncovalently bound complexes was deduced by their molecular weights (Table II) and component fragments (Table I). Protein migrating as band 1 dissociated in SDS (Fig. 2) into components of 195,000 (DD) and 45-55,000 (E), confirming its identity as $\mathrm{DD} / \mathrm{E}(9,10)$. Complex 2 had a molecular weight approximately double that of $\mathrm{DD} / \mathrm{E}$ (Table II) and dissociated into fragments DY, DD, and E (Fig. 2).
The proposed structure of complex 2 is shown in Fig. 6, in which fibrin is represented as a two-stranded, half-staggered overlap fiber, with noncovalent attractions between $\mathrm{D}$ and $\mathrm{E}$ domains of adjacent fibrin strands $(48,49)$. Whereas complex 1 would derive from fragment DD of one fibrin strand and fragment $E$ from the adjacent strand, joined by noncovalent attraction, complex 2 would consist of two antiparallel DY fragments, held together by the same noncovalent bond between adjacent DD and $E$ regions. The structure of complex 2 is compatible with the measured molecular weight, since it is double that of complex 1 and within $6 \%$ of the sum of two component YD fragments of 247,000 each (Fig. 2). Cleavage between the D and $E$ 


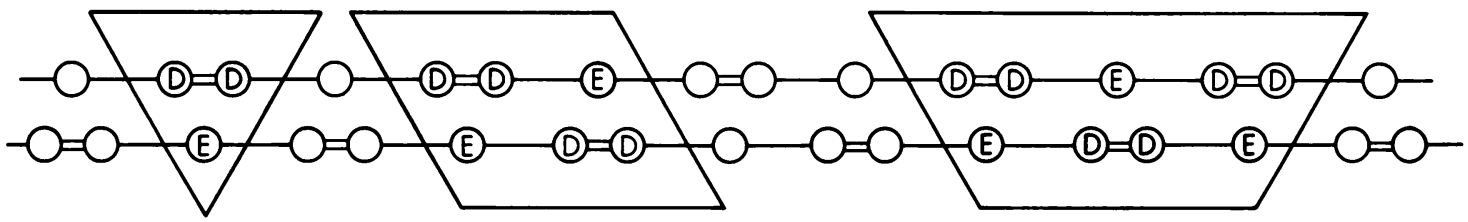

(E)

(YD)

(YY)

\title{
Complex 1
}

Complex 2

Complex 3

\begin{abstract}
Figure 6 Structure and in situ derivation of complexes 1, 2, and 3 derived from plasmic degradation of crosslinked fibrin. Two linear fibrin polymers are shown in a half-staggered overlap array with covalent crosslinks between $\gamma$ chains indicated as double lines between adjacent $D$ domains. The crosslinks between $\alpha$ chains are not shown. Complex 1 shows D dimer (DD) apposed to fragment $E$ of the other fibrin strand. The nomenclature of the covalently-bound fragments contributed by each fibrin strand to complexes 2 and 3 corresponds to that indicated in Fig. 5 . The complexes could therefore be expressed as DD/E (complex 1), DY/YD (complex 2), and DXD/YY (complex 3). All complexes that are larger than complex 1 have considerable heterogeneity resulting from proteolytic cleavages that do not disrupt the noncovalent bonds holding the fragments of the two chains together as a complex.
\end{abstract}

domains of one fibrin strand of complex 2 would result in potential DD and E fragments, as seen in Fig. 2, but would not disrupt its noncovalently bound structure. Plasmic cleavages between DD and E domains in both adjacent fibrin polymer strands would result in two complex 1 units.

The molecular weight of complex 3 was only $3 \%$ greater than three times that of complex 1. Two-dimensional electrophoresis (Fig. 2B, Table I) showed that it contained covalently bound fragments as large as DXD $(461,000)$ and YY $(285,000)$. Its molecular weight measured by equilibrium sedimentation $(703,000)$ was within $6 \%$ of the sum $(746,000)$ of its intact constituent subunits, DXD and YY. Cleavages between D and E domains within the complex could produce fragments DY and DD observed in Fig. 2, without necessarily disrupting its noncovalently bound integrity.

The components of complex 4 were not well resolved by two-dimensional electrophoresis and its structure was less well determined. The logical extension of the scheme in Fig. 6 would suggest an alignment of antiparallel, noncovalently bound fragments YXD and DXY. The molecular weight of such a complex should be four times that of complex $1(912,000)$ or equal to the sum of its component fragments $(1,000,000)$. The observed molecular weight of 850,000 was $7 \%$ and $15 \%$ less than the respective predicted maximal sizes. This suggests that complex 4 was an unstable derivative or that it was seldom released from fibrin in its intact form.

Our scheme of complexes is the only series of twostranded noncovalently bound derivatives that could result from plasmin cleavages between complementary $D$ and $E$ domains. Furthermore, variations on the proposed basic structure of the complexes can be logically drawn to explain the multitude of component fragments that dissociate in SDS from the complexes (Fig. 2). For instance, an adjacent $\mathrm{D}$ or $\mathrm{E}$ domain may be attached to the complex as the result of cleavage just outside the usual boundary of the complex. Examples of such variants were DY in complex 1 , YY in complex 2 , and DXY in complex 3.

Internal cleavages between $\mathrm{D}$ and $\mathrm{E}$ domains were probably frequent, as indicated by the large amounts of DD in complex 2 and of DY and DD in complex 3. Variations resulting from cleavages at or near a $\gamma \gamma$ isopeptide bond occurred less often but resulted in $D$ and $\mathrm{Y}$ in complex 1 and in $\mathrm{Y}, \mathrm{D}$, and $\mathrm{XD}$ in complex 2. Relatively large amounts of fragments $\mathrm{X}, \mathrm{Y}$ and $\mathrm{XD}$ were present in complex 3 (Fig. 2), indicating that the $\gamma \gamma$ cleavage site may be more accessible within the intact fibrin strand than in liberated complexes.

Our results are in conflict with those of Gaffney and Joe (13) who suggest that digestion of crosslinked fibrin by plasmin does not yield a variety of high molecular weight fragments, but only $\mathrm{DD} / \mathrm{E}$ which may then aggregate to form larger complexes. Although our data do not rule out complex aggregation subsequent to release into solution, the structure of component covalent fragments making up the complexes are not compatible with their derivation by noncovalent aggregation of DD/E complexes. Our results support the conclusions of Reganon et al. (12) that DY and YY are the next larger covalent fragments after DD, although our measured molecular weights differ.

The data presented supports the two-stranded halfstaggered overlap model (15) as the basic structure of crosslinked fibrin. The principal cleavage site for crosslinked fibrin is apparently the same as for fibrinogen, namely in the "coiled coil" region connecting the central and terminal domains (50). The complexes larger than DD/E probably have considerable physio- 
logic relevance, inasmuch as they always comprise the bulk of newly released protein from fibrin clots undergoing plasmic degradation in vitro (14).

\section{ACKNOWLEDGMENTS}

This work was supported in part by grants HL-07151 and HL-18208 from the National Heart, Lung and Blood Institute of the National Institutes of Health, Bethesda,Md.

\section{REFERENCES}

1. Marder, V. J., N. R. Shulman, and W. R. Carroll. 1967. The importance of intermediate degradation products of fibrinogen in fibrinolytic hemorrhage. Trans. Ass. Amer. Phys. 53: 156-167.

2. Marder, V. J., N. R. Shulman, and W. R. Carroll. 1969. High molecular weight derivatives of human fibrinogen produced by plasmin. I. Physicochemical and immunological characterization. J. Biol. Chem. 244: 21112119.

3. Nussenzweig, V., M. Seligmann, U. Pelmont, and P. Grabar. 1960. Les produits de dégradation du fibrinogène humain par la plasmine. I. Séparation et propriétés physico-chimiques. Ann. Inst. Pasteur (Paris). 100: 377389.

4. Nussenzweig, V., M. Seligmann, and P. Grabar. 1961. Les produits de dégradation du fibrinogène humain par la plasmine. II. Étude immunologique: mise en évidence d'anticorps anti-fibrinogène natif possédant des spécificités différentes. Ann. Inst. Pasteur (Paris). 100: 490-508.

5. Ferguson, E. W., L. J. Fretto, and P. A. McKee. 1975. A re-examination of the cleavage of fibrinogen and fibrin by plasmin. J. Biol. Chem. 250: 7210-7218.

6. Gaffney, P. J., and M. Brasher. 1973. Subunit structure of the plasmin-induced degradation products of crosslinked fibrin. Biochim. Biophys. Acta. 295: 308-313.

7. Kopeć, M., E. Teisseyre, G. Dudek-Wojciechowska, M. Kloczewiak, A. Pankiewicz, and Z. S. Latallo. 1973. Studies on the "Double D" fragment from stabilized bovine fibrin. Thromb. Res. 2: 283-291.

8. Pizzo, S. V., L. M. Taylor, Jr., M. L. Schwartz, R. L. Hill, and P. A. McKee. 1973. Subunit structure of Fragment D from fibrinogen and crosslinked fibrin. J. Biol. Chem. 248: 4584-4590.

9. Hudry-Clergeon, G., L. Paturel, and M. Suscillon. 1974. Identification d'un complexe (D-D) . . . E dans les produits de degradation de la fibrine bovine stabilisee par la Facteur XIII. Pathol. Biol. 22(Suppl.): 47-52.

10. Gaffney, P. J., D. A. Lane, V. V. Kakkar, and M. Brasher. 1975. Characterisation of a soluble $D$ dimer $-E$ complex in crosslinked fibrin digests. Thromb. Res. 7: 89-99.

11. Alkjaersig, N., A. Davies, and A. Fletcher. 1977. Fibrin and fibrinogen proteolysis products: comparison between gel filtration and SDS polyacrylamide electrophoresis analysis. Thromb. Haemostasis. 38: 524-535.

12. Reganon, E., V. Vila, and J. Aznar. 1978. Identification of high molecular weight derivatives of plasmic digests of cross-linked human fibrin. Thromb. Haemostasis. 40: 368-376.

13. Gaffney, P. J., and F. Joe. 1979. The lysis of crosslinked human fibrin by plasmin yields initially a single molecular complex, D dimer-E. Thromb. Res. 15: 673-687.

14. Francis, C. W., V. J. Marder, and S. E. Martin. 1980. Plasmic degradation of crosslinked fibrin. I. Structural analysis of the particulate clot and identification of new macromolecular soluble complexes. Blood. 56: 456-464.
15. Ferry, J. D. 1952. The mechanism of polymerization of fibrin. Proc. Natl. Acad. Sci. U. S. A. 38: 566-569.

16. Krakow, W., G. F. Endres, B. M. Siegel, and H. A. Scheraga. 1972. An electron microscopic investigation of the polymerization of bovine fibrin monomer. J. Mol. Biol. 71: 95-103.

17. Doolittle, R. F. 1973. Structural aspects of the fibrinogenfibrin conversion. Adv. Protein. Chem. 27: 1-109.

18. Hermans, J. 1979. Models of fibrin. Proc. Natl. Acad. Sci. U. S. A. 76: 1189-1193.

19. Gaffney, P. J. 1975. Distinction between fibrinogen and fibrin degradation products in plasma. Clin. Chim. Acta. 65: 109-115.

20. Lane, D. A., F. E. Preston, M. E. VanRoss, and V. V. Kakkar. Characterization of serum fibrinogen and fibrin fragments produced during disseminated intravascular coagulation. Br. J. Haematol. 40: 609-615.

21. Graeff, H., R. Hafter, and R. von Hugo. 1977. Molecular aspects of defibrination in a case of amniotic fluid embolism. Thromb. Haemostasis. 38: 724-727.

22. Francis, C. W., V. J. Marder, and S. E. Martin. 1979. Detection of circulating crosslinked fibrin derivatives by a heat extraction-SDS gradient gel electrophoretic technique. Blood. 54: 1282-1295.

23. Plow, E. F., and T. S. Edgington. 1973. Discriminating neonatigenic differences between fibrinogen and fibrin derivatives. Proc. Natl. Acad. Sci. U. S. A. 70: 11691173.

24. Budzynski, A. Z., V. J. Marder, M. E. Parker, P. Shames, B. S. Brizuela, and S. A. Olexa. 1979. Antigenic markers of Fragment DD, a unique plasmic derivative of human crosslinked fibrin. Blood. 54: 1282-1295.

25. Johnson, A. J., D. L. Kline, and N. Alkjaersig. 1969. Assay methods and standard preparations for plasmin, plasminogen and urokinase in purified systems. Thromb. Diath. Haemorrh. 21: 259-272.

26. Davis, B. J. 1964. Disc electrophoresis. II. Method and application to human serum proteins. Ann. N. Y. Acad. Sci. 121: 404-427.

27. Margolis, J., and K. G. Kenrick. 1967. Polyacrylamide gelelectrophoresis across a molecular sieve gradient. Nature (Lond.). 214: 1334-1336.

28. Neville, D. M., Jr. 1971. Molecular weight determination of protein-dodecyl sulfate complexes by gel electrophoresis in a discontinuous buffer. J. Biol. Chem. 246: 6328-6334.

29. Martin, S. E., V. J. Marder, C. W. Francis, L. S Loftus, and G. H. Barlow. 1980. Enzymatic degradation of the Factor VIII-von Willebrand protein: a unique tryptic fragment with ristocetin cofactor activity. Blood. 55: 848858.

30. Schachman. J. 1959. Ultracentrifuge in Biochemistry. Academic Press, Inc. New York.

31. Schachman, H. K., and S. J. Edelstein. 1966. Ultracentrifuge studies with absorption optics. IV. Molecular weight determinations at the microgram level. Biochemistry. 5: 2681-2705.

32. Blombäck, B., and A. J. Johnson. 1971. Joint report of the subcommittee on nomenclature and on fibrinolysis, thrombolysis and intravascular coagulation. Thromb. Diath. Haemorrh. 51(Suppl.): 251-256.

33. Marder, V. J., and A. Z. Budzynski. 1975. Data for defining fibrinogen and its plasmic degradation products. Thromb. Diath. Haemorrh. 33: 199-207.

34. Francis, C. W., V. J. Marder, and S. E. Martin. 1980. Demonstration of a large molecular weight variant of the $\gamma$ chain of normal human plasma fibrinogen. J. Biol. Chem. 255: 5599-5604. 
35. Marder, V. J., A. Z. Budzynski, and G. H. Barlow. 1976. Comparison of the physico-chemical properties of Fragment $D$ derivatives of fibrinogen and Fragment D-D of crosslinked fibrin. Biochim. Biophys. Acta. 427: 1-14.

36. Chen, R., and R. F. Doolittle. 1971. $\gamma-\gamma$ cross-linking sites in human and bovine fibrin. Biochemistry. 10: 4486-4491.

37. Mills, D., and S. Karpatkin. 1970. Heterogeneity of human fibrinogen: possible relation to proteolysis by thrombin and plasmin as studied by SDS-polyacrylamide gel electrophoresis. Biochem. Biophys. Res. Commun. 40: 206-211.

38. Gaffney, P. J., and P. Dobos. 1971. A structural aspect of human fibrinogen suggested by its plasmin degradation. FEBS (Fed. Eur. Biochem. Soc.) Lett. 15: 13-16.

39. Pizzo, S. V., M. L. Schwartz, R. L. Hill, and P. A. McKee. 1972. The effect of plasmin on the subunit structure of human fibrinogen. J. Biol. Chem. 247: 636-645.

40. Furlan, M., and E. A. Beck. 1972. Plasmic degradation of human fibrinogen. I. Structural characterization of degradation products. Biochim. Biophys. Acta. 263: 631-644.

41. Mills, D. A. 1972 . Molecular mode for the proteolysis of human fibrinogen by plasmin. Biochim. Biophys. Acta. 263: 619-630.

42. Pizzo, S. V., M. L. Schwartz, R. L. Hill, and P. A. McKee. 1973. The effect of plasmin on the subunit structure of human fibrin. J. Biol. Chem. 248: 4574-4583.

43. Budzynski, A. Z., V. J. Marder, and J. R. Shainoff. 1974. Structure of plasmic degradation products of human fibrinogen. Fibrinopeptide and polypeptide chain analysis. J. Biol. Chem. 249: 2294-2302.

44. Takagi, T., and R. F. Doolittle. 1975. Amino acid sequence studies on plasmin-derived fragments of human fibrinogen: amino terminal sequences of intermediate and terminal fragments. Biochemistry. 14: 940-946.

45. Takagi, T., and R. F. Doolittle. 1975. Amino acid sequence of the carboxy-terminal cyanogen bromide peptide of the human fibrinogen $\beta$-chain: Homology with the corresponding $\gamma$-chain peptide and presence in Fragment $D$. Biochim. Biophys. Acta. 386: 617-622.

46. Olexa, S. A., A. Z. Budzynski, and V. J. Marder. 1979. Chemical modification of high molecular weight plasmic degradation products of human crosslinked fibrin. Biochim. Biophys. Acta. 576: 39-50.

47. Purves, L. R., G. G. Lindsey, G. Brown, and J. Franks. 1978. Stabilization of the plasmin digestion products of fibrinogen and fibrin by calcium ions. Thromb. Res. 12: 473-484.

48. Heene, D. L., and F. R. Matthias. 1973. Adsorption of fibrinogen derivatives on insolubilized fibrinogen and fibrin. Thromb. Res. 2: 137-154.

49. Kudryk, B. J., D. Collen, K. R. Woods, and B. Blombäck 1974. Evidence for localization of polymerization sites in fibrinogen. J. Biol. Chem. 249: 3322-3325.

50. Doolittle, R. F., D. M. Goldbaum, and L. R. Doolittle. 1978. Designation of sequences involved in the "coiledcoil" interdomainal connections in fibrinogen: construction of an atomic scale model. J. Mol. Biol. 120: 311-325. 